\title{
Difusão de Íons Cloreto em Cimentos de Escória de Alto-Forno e Pozolânico
}

\author{
Diffusion of chloride ions in blast-furnace slag and pozolan cements
}

\author{
Tiago Agra 1 \\ Eduardo Lobo ${ }^{2}$ \\ Eliana Monteiro ${ }^{1}$ \\ ${ }^{1}$ Escola Politécnica de Pernambuco, Universidade de Pernambuco, Recife, Brasil \\ E-mail do autor principal: Tiago Agra tiagoagraengenharia@gmail.com
}

\section{Resumo}

A escolha do tipo do cimento constitui uma etapa importante para durabilidade das obras de construção civil, pois cada tipo dispõe de propriedades específicas que podem indicar melhor eficácia no combate às ações deletérias. A corrosão prematura das armaduras do concreto armado é um problema evidenciado em todo mundo, trazendo elevados custos de manutenção/recuperação, sendo a contaminação por cloreto um dos principais efeitos causadores. A revisão bibliográfica mostra, de modo geral, que os cimentos com adição de escórias e pozolanas, em longo prazo, são mais duráveis e vantajosos, pois são mais compactos, fator que influencia diretamente na redução das vias de penetração, possibilitando, potencialmente, melhor desempenho em obras que ficarão expostas à ação de cloretos. Este trabalho apresenta os resultados da difusibilidade de íons cloreto e resistência à compressão do cimento Portland de Alto Forno (CP III) e Pozolânico (CP IV), de mesma classe de resistência, com o objetivo de verificar a influência das adições frente à facilidade de penetração dos íons cloreto no concreto. Com relação à difusibilidade dos íons cloretos, os cimentos CP III e CP IV apresentaram valores de carga passante (Coulombs) de 1248 C e 4643 C, sendo classificados de baixa e elevada penetração aos íons cloreto, respectivamente. Em suma, abstraindo-se de outras características não estudadas, a amostra de cimento CP III apresentou melhor contribuição para a durabilidade do concreto quando comparada com a amostra de CP IV, em igualdade de condições.

Palavras-Chave: Corrosão; Difusão de Ions Cloreto; Durabilidade; Adições em Cimentos.

\begin{abstract}
The choice of cement type is an important step for the durability of civil construction building, since each type has specific properties that may indicate better effectiveness in combating harmful actions. Premature corrosion of reinforced concrete steel is a worldwide problem, bringing to high spending maintenance /recovery, with chloride contamination being one of the main causative effects. The bibliographic references, in general, that the cements with addition of slag and pozzolans, in the long term, are more durable and advantageous, because they are more compact, a factor that directly influences the reduction of the penetration course, better performance in works that will be exposed to the action of chlorides. This work presents the results of the diffusibility of chloride ions and compressive strength of blast-furnace slag (CP III) and Pozzolanic (CP IV) Portland cements, of the same resistance class, in order to verify the influence of the additions with respect to the facility Penetration of the chloride ions in the concrete. In relation to the diffusibility of chloride ions, the CP III and CP IV cements showed throughput values (Coulombs) of 1248 C and 4643 C, being classified as low and high penetration to chloride ions, respectively. In summary, apart from other characteristics not studied, the cement sample CP III presented a better contribution to the durability of the concrete when compared to the sample of CP IV, in equal conditions.
\end{abstract}

Key-words: Corrosion; Diffusion of Chloride Ions; Durability; Additions in Cement.

122

http: / / dx.doi.org/10.25286/repa.v2i2.569 


\section{Introdução}

\subsection{Contextualização do Problema}

As abordagens modernas não baseiam apenas, de forma simplista, na resistência à compressão como único indicador de durabilidade. Elas se atêm, atualmente, as características e propriedades do material especificadas em função de uma vida útil almejada considerando determinado ambiente [4].

Em virtude da observação de diversos casos de deterioração prematura, por carência ou falta de estudo, é de extrema importância a busca de melhorias no desempenho das obras e verificação do comportamento frente aos agentes causadores. O conhecimento adquirido há mais de um século, além da experiência com as construções já executadas, mostra que, sempre que o estado da arte é respeitado, as obras em concretos são extremamente duráveis e resistentes ás agressões do meio ambiente [16].

O crescente aumento de estruturas com manifestações patológicas precoce, impõe a necessidade de melhorar o planejamento em projetos, fiscalização, execução e manutenção, pois o alto custo de reparo/recuperação, e os transtornos causados, determinam a inevitabilidade de projetar visando a durabilidade. A compreensão dos mecanismos e das manifestações patológicas, possibilitam o planejamento mais eficaz na elaboração de estruturas com melhor interação com meio, levando em consideração ações especificas delimitadoras de cada obra. Nenhum material é propriamente durável. Como resultado de interações ambientais, a microestrutura e, consequentemente, suas propriedades mudam com o tempo. O material atinge sua vida útil quando suas propriedades, sob determinadas condições de uso a capacidade de resistir [14].

A corrosão das armaduras promove expansão das barras, reduzindo sua seção efetiva, ocasionando a demolição do concreto invólucro, refletindo simultaneamente no comportamento mecânico da estrutura podendo leva-la ao colapso. O mecanismo de corrosão que causa maiores prejuízos e, portanto, o mais estudado, é a corrosão por ação de cloretos, que tem como principais fontes de contaminação: água de amassamento, aditivos, agregados contaminados, e a penetração de soluções de sais de degelo ou de água do mar [7]. O mercado mundial de recuperação e manutenção estrutural do concreto armado vem crescendo e exigindo, segundo Ribeiro [19], a criação de novas soluções, para minimização dos problemas e diminuição nos custos de reparo.
Os tipos de cimento/adições estão completamente atrelados ao bom funcionamento estrutural, em função de sua composição química, pois podem oferecer eficiência diversas com relação aos requisitos de desempenho e durabilidade das obras. A reatividade das pozolanas e escórias, contidas nos cimentos em estudo, se processa após algumas semanas, reforça os efeitos de hidratação do clínquer, conduzindo, em logo prazo a uma porosidade de mesma ordem de grandeza, porém, diminui o tamanho dos poros maiores, o que é favorável no que tange à durabilidade [8].

Buscando o desenvolvimento sustentável para prolongar a vida útil das estruturas, redução dos custos de manutenção e prevê os riscos de degradação, o presente trabalho visa a análise da influência das adições minerais, escória de alto-forno e pozolana, de cimentos comerciais, na velocidade da difusão dos cloretos em concreto para a compreensão do aglomerante que ofereça melhores propriedades frente à ação prejudicial da penetração de cloretos. Foram realizados ensaios de resistência mecânica à compressão, NBR 5739 [1] e teste de indicação elétrica para medição da capacidade do concreto de resistir à penetração de íon cloreto, ASTM C1202 [3].

\subsection{Cimentos}

Nas décadas anteriores, o conhecimento na área tecnológica do cimento era reduzido, assim como a compreensão dos mecanismos de deterioração. A escolha era realizada de maneira aleatória, buscando apenas resistência e menor custo, desconsiderando a sinergia do material com os diversos microclimas. Com o avanço da tecnologia foi possível o aprimoramento dos materiais de construção, em especial o cimento, exigindo que suas propriedades fossem conhecidas para aplicação de modo a satisfazer a necessidade de mercado. A indústria brasileira disponibiliza atualmente diversos tipos de cimento, que são classificados em função de sua composição, conforme Tabela 1, que mostra os tipos de cimentos comerciais mais vendidos no país.

Os cimentos que possuem maiores teores de adição são o CPIII (entre 35\% a 70\% de escória) e o CPIV (entre $15 \%$ a $50 \%$ de pozolanas). 
Tabela 1: Componentes do cimento.

Fonte: ABNT NBR.

\begin{tabular}{|l|c|c|c|c|c|c|}
\cline { 3 - 7 } & \multirow{2}{*}{} & \multirow{2}{*}{ Norma } & \multicolumn{5}{|c|}{ Componentes (\% massa) } \\
\cline { 3 - 7 } & & \multicolumn{2}{|c|}{ Clínquer + Gesso } & \multicolumn{3}{c|}{ Adições } \\
\cline { 3 - 7 } & & Limite inferior & Limite superior & Carbonáticas & Escória & Pozolana \\
\hline CP I & NBR 5732 & $100 \%$ & $100 \%$ & $0 \%$ & $0 \%$ & $0 \%$ \\
\hline CP I S & NBR 5733 & $95 \%$ & $99 \%$ & - & - & $1 \%-5 \%$ \\
\hline CP II-E & NBR 11578 & $56 \%$ & $94 \%$ & $0 \%-10 \%$ & $6 \%-34 \%$ & - \\
\hline CP II-F & NBR 11578 & $90 \%$ & $94 \%$ & $6 \%-10 \%$ & - & - \\
\hline CP II-Z & NBR 11578 & $86 \%$ & $94 \%$ & $0 \%-10 \%$ & - & $6 \%-14 \%$ \\
\hline CP III & NBR 5735 & $25 \%$ & $65 \%$ & $0 \%-5 \%$ & $35 \%-70 \%$ & - \\
\hline CP IV & NBR 5736 & $45 \%$ & $85 \%$ & $0 \%-5 \%$ & - & $15 \%-50 \%$ \\
\hline CP V (ARI) & NBR 5733 & $95 \%$ & $100 \%$ & $0 \%-5 \%$ & - & - \\
\hline
\end{tabular}

\subsection{Revisão Bibliográfica}

O estudo sobre a difusão de cloretos pela pasta de cimento desperta grande atenção porque tem relação direta com a facilidade de penetração dos íons no concreto. Para dosagens de concreto que são normalmente empregadas na prática, o teor crítico de cloretos que inicia a corrosão se encontra na ordem de $0,4 \%$ em relação ao consumo de cimento. Entretanto, esse valor crítico depende de fatores como: grau de carbonatação, qualidade do concreto e umidade relativa [14]. Além disso, a difusão é maior quando aumentamos a relação a/c e o tipo da adição do cimento, pois tem relação direta com a frequência de poros contínuos com diâmetros maiores [8].

Os cloretos livres, ou também cloretos solúveis em água, dissolvidos nos poros, são aqueles que ficam sob a forma iônica, de fácil mobilidade, já o fixo é quimicamente ligado à matriz cimentícia, formando os cloroaluminatos. A quantidade total de cloretos no concreto é a soma dos cloretos livres e fixos.

Martín-Pérez et al., [11], considera que apenas os cloretos livres são os responsáveis por iniciar o processo de corrosão, pois somente neste estado seria possível sua penetração pelo cobrimento do concreto, entretanto outros autores, Camacho et al. [5], Liu et al. [10] e Meijers et al. [15], salientam que os cloretos combinados aumentam a reserva de íons disponível em contato com o aço, podendo ser liberados por processos como a redução do pH. Assim, a capacidade dos produtos da hidratação do cimento, principalmente do $\mathrm{C} 3 \mathrm{~A}$, de combinar-se com íons cloreto, formam o cloroaluminato, tem um efeito importante na difusão, removendo íons da solução dos poros e desacelerando a taxa de penetração.

Camacho et al. [5] analisou que a carbonatação tem influência negativa sobre o coeficiente de difusão dos cloretos, funcionando como um "caminho privilegiado" para a penetração dos mesmos. J. Liu et al. [9] analisou que na medida em que ocorre a carbonatação de estruturas contaminadas por cloretos, pode ocorrer também o aumento de cloretos livres devido à instabilidade dos cloroaluminatos promovida pela redução do $\mathrm{pH}$ no interior do concreto.

Crauss [6] estudou concretos de mesma classe de resistência confeccionados com CPIV, CPII-F e CPV (porém não comparou com o CPIII, verificando que frente à penetração de cloreto, o cimento pozolânico ofereceu menor penetração do íon devido ao refinamento dos poros promovidos pela adição pozolânica, em sua composição, verificando também que na medida em que aumentamos a resistência mecânica, é reduzida sua capacidade de penetração. Pereira [18] analisou a capacidade de proteção de alguns cimentos (CPII-Z, CPIII e CPIV), em relação à corrosão por íons cloretos através do ensaio de potencial de corrosão e absorção capilar. Nessa avaliação os resultados evidenciaram melhor desempenho do cimento CP III, pois apresentou menor corrosão por íons cloreto e redução da absorção capilar. Além disso, Pereira [17] observou que à proporção que se diminui a relação $a / c$, maior será a proteção proporcionada à armadura, devido à diminuição do tamanho dos poros e, por 
consequência, maior será a resistência à penetração de fluídos e agentes agressivos.

Segundo Barochel-bouny, Capra e Laurens [4], de modo geral, os concretos preparados com adições minerais (escória de alto-forno, metacaulim, cinza volante, sílica ativa, fíleres etc.) limitam, a difusão de íons cloreto. Medeiros Junior [12] em seu estudo com cimentos comerciais, mostrou, em seu estudo, que os que possuem maiores teores de adições, CPIII (65\% de escória de alto-forno) e CPIV (35\% de pozolana), contribuíram mais para elevar a resistividade elétrica do concreto, além de promover, ao longo do tempo, evolução na redução da porosidade devido à progressiva hidratação dos compósitos cimentícios. Sabe-se que as escórias e pozolanas refinam os poros do concreto e reduzem a permeabilidade e mobilidade dos íons dentro da microestrutura do concreto [13]. Pereira [18] estudou concretos com incorporação de escória de alto-forno, em substituição parcial do cimento Portland, e verificou que são mais eficientes em prevenir o ingresso de íons cloretos, comparados com os produzidos com outros cimentos.

\section{Resultados e Discussão}

\subsection{Caracterização dos Materiais de Partida}

A distribuição percentual da composição dos componentes em massa dos cimentos em estudo, pode ser observada na Tabela 2 . Os resultados dos ensaios de caracterização no estado fresco e endurecido são apresentados na Tabela 3. O teor de argamassa foi fixado em $50,5 \%$, com valor de relação água/cimento igual a 0,50.

Tabela 2: Composição \% em massa dos cimentos em estudo.

\begin{tabular}{|c|c|c|c|c|}
\hline \multicolumn{2}{|c|}{ Norma } & NBR 5735 & NBR 5736 \\
\hline \multirow{4}{*}{$\begin{array}{c}\text { (\%) } \\
\text { massa })\end{array}$} & \multirow{2}{*}{ Clínquer + Gesso } & $45 \%$ & $50 \%$ \\
\cline { 2 - 4 } & \multirow{3}{*}{ Adições } & Carbonáticas & $5 \%$ & $5 \%$ \\
\cline { 3 - 5 } & & Escória & $50 \%$ & - \\
\cline { 3 - 5 } & Pozolana & - & $45 \%$ \\
\hline
\end{tabular}

Procedeu-se, posteriormente, a moldagem de corpos de prova cilíndricos $(10 \times 20 \mathrm{~cm})$ e cura, NBR5738 [3], para realização dos seguintes ensaios no estado endurecido:
- Determinação da resistência à compressão nas idades de 7 e 28 dias;

- Determinação da penetração de íons cloreto aos 28 dias;

Tabela 3: Características da argamassa da pesquisa.

\begin{tabular}{|c|c|c|c|}
\hline \multicolumn{2}{|l|}{ Parâmetros } & CP III & CP IV \\
\hline \multirow{6}{*}{ 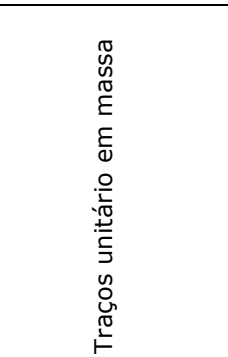 } & Cimento & 1 & 1 \\
\hline & Areia de Quartzo & 1,204 & 1,204 \\
\hline & Areia artificial & 0,802 & 0,802 \\
\hline & Pedrisco & 0,884 & 0,884 \\
\hline & Brita 1 & 2,061 & 2,061 \\
\hline & Água & 0,5 & 0,5 \\
\hline \multicolumn{2}{|c|}{ Aditivo Plastificante (\%) } & 1,2 & 1,2 \\
\hline \multicolumn{2}{|c|}{ Consumo $\left(\mathrm{Kg} / \mathrm{m}^{3}\right)$} & 363 & 363 \\
\hline \multicolumn{2}{|c|}{ Teor de Argamassa (\%) } & 50,5 & 50,5 \\
\hline \multicolumn{2}{|l|}{ Umidade (\%) } & 8,38 & 8,38 \\
\hline \multicolumn{2}{|c|}{ Massa específica $\left(\mathrm{Kg} / \mathrm{m}^{3}\right)$} & 2420 & 2370 \\
\hline \multicolumn{2}{|l|}{ Teor de $\operatorname{Ar}(\%)$} & 1,2 & 0,9 \\
\hline \multicolumn{2}{|c|}{ Abatimento $(\mathrm{mm})$} & 145 & 150 \\
\hline \multirow{2}{*}{$\begin{array}{c}\text { Resistência à } \\
\text { compressão (Mpa) }\end{array}$} & 7 dias & 36,2 & 41,2 \\
\hline & 28 dias & 52,1 & 50,4 \\
\hline
\end{tabular}

Logo após a moldagem, os corpos de prova foram colocados em câmara úmida e desmoldados após $24 \mathrm{~h}$ e mantidos em cura até a idade de ensaio.

\subsection{Difusibilidade de Íons Cloreto}

Para investigar o comportamento dos concretos confeccionados frente à difusibilidade dos íons cloreto, adotou-se o método [3] - "Standard Test Method for Eletrical Indication of Concrete's Ability to Resist Chloride Ion Penetration", sendo as medidas realizadas após cura de 28 dias. A difusão de cloretos tem sido investigada também como parâmetro da durabilidade pelo fato de serem íons pequenos que indicariam a maior ou menor vulnerabilidade do concreto aos agentes agressivos em geral. $O$ procedimento para medir a difusão dos cloretos consiste em submeter uma parte amostra de concreto com $5 \mathrm{~cm}$ de altura e $10 \mathrm{~cm}$ de diâmetro (extraída no 
terço médio de um corpo de prova cilíndrico de $10 \mathrm{~cm}$ de diâmetro e $20 \mathrm{~cm}$ de altura) à passagem de corrente elétrica, por um período de $6 \mathrm{~h}$, sob uma diferença de potencial de $60 \mathrm{~V}$. O corpo de prova tem uma das extremidades imersas em solução de hidróxido de sódio e a outra em solução de cloreto de sódio, conforme ilustrado nas Figuras 1 e 2 . A carga total passante é relacionada com a resistência do concreto à penetração de íons cloretos.

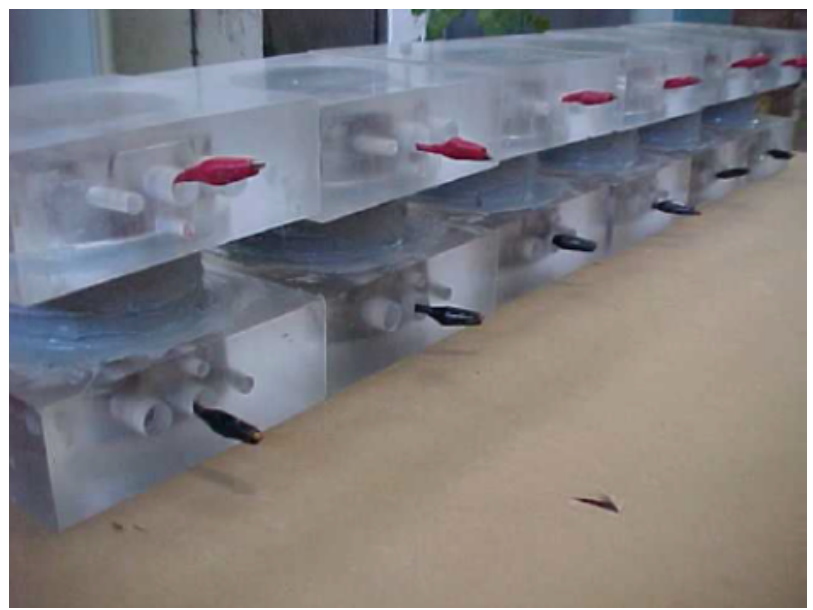

Figura 1: Extremidades das amostras imersas em solução de hidróxido de sódio e cloreto de sódio.

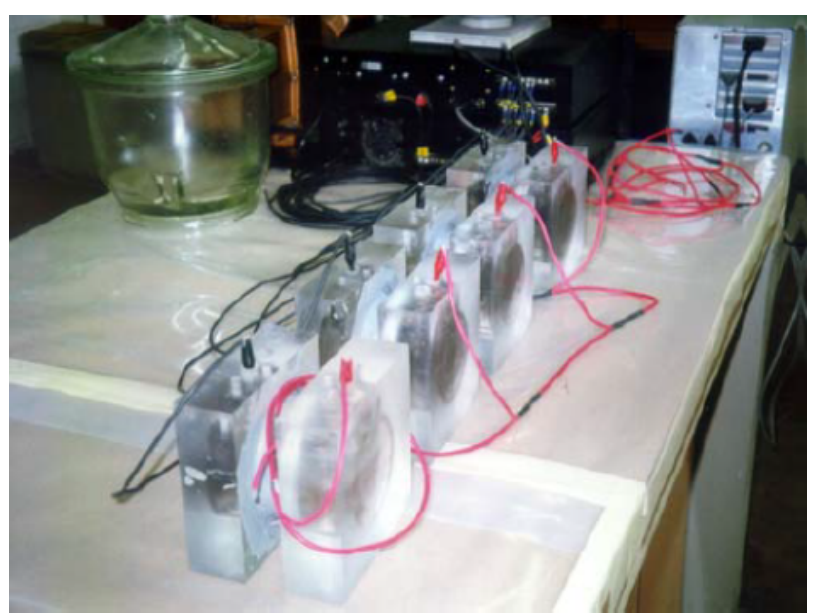

Figura 2: Amostras submetidas à passagem de corrente elétrica por $6 \mathrm{~h}$.

Os resultados obtidos são semi-quantitativos, tendo em vista a amplitude das faixas classificatórias apresentadas na ASTM C 1202 [3], Tabela 4. 126
Os resultados de difusibilidade de íons cloreto obtidos com os corpos de prova moldados com os cimentos CP III RS e CP IV, na idade de 28 dias, estão apresentados na Tabela 5.

Tabela 4: Resultados da difusão dos íons cloreto.

\begin{tabular}{|c|c|c|}
\cline { 2 - 3 } \multicolumn{1}{c|}{} & CP III & CP IV \\
\hline $\begin{array}{c}\text { Penetração Íons } \\
\text { Cloreto Carga (C) }\end{array}$ & 1248 & 4643 \\
\hline Classificação & Baixa & Elevada \\
\hline
\end{tabular}

\section{Conclusões}

Em suma, abstraindo-se de outras características não estudadas, a amostra de cimento CP III apresentou melhor contribuição para a durabilidade do concreto quando comparada com a amostra de CP IV-32, em igualdade de condições. Através dos resultados experimentais sobre à difusão de íons cloreto em cimentos com escória de alto-forno e pozolânicos, podem-se extrair a seguinte conclusão:

- Os cimentos CP III e CP IV apresentaram valores de carga passante (Coulombs) de 1248 C e 4643 C, sendo classificados de baixa e elevada penetração aos íons cloreto, respectivamente. Esses valores mostram que os cimentos com escória de alto-forno são mais eficientes em resistir ao ingresso de íons cloretos, pois são menos porosos e mais duráveis, devido à maior colmatação dos poros. As escórias refinam melhor os poros, propiciando ao concreto redução da permeabilidade e mobilidade dos íons dentro de sua microestrutura.

\section{Referências}

[1] ASSOCIAÇÃO BRASILEIRA DE NORMAS TÉCNICAS (ABNT). NBR 5739 - Concreto - Ensaio de Compressão de Corpos-de-prova Cilíndricos, 2007.

[2] ASSOCIAÇÃO BRASILEIRA DE NORMAS TÉCNICAS (ABNT). NBR 5738 - Concreto -

http://dx.doi.org/10.25286/repa.v2i2.569 
Procedimento para moldagem e cura de corposde-prova, 2015.

[3] ASTM C1202. Standard Test Method for Electrical Indication of Concrete's Ability to Resist Chloride Ion Penetration, 2010.

[4] BAROCHEL-BOUNY, V.; CAPRA, B.; LAURENS, S.. A durabilidade das armaduras e do concreto de cobrimento. In: OLLIVIER, J.P.; VICHOT, A, 2014. Durabilidade do Concreto: Bases Científicas para a formulação de concretos duráveis de acordo com o ambiente. Tradução: O. Cascudo e H. Carasek. São Paulo: IBRACON, 2014. p. 255-326.

[5] CAMACHO, Gustavo; MALHEIRO, Raphaele; CAMÕES Aires; MEIRA Gibson. Ação Combinada da Carbonatação e dos Iões Cloreto em Betões com Cinzas Volantes. In: II congresso luso-brasileiro de materiais de construção sustentáveis, 2016, João Pessoa, Brasil.

[6] CRAUSS, C.. Penetração de cloretos em concretos com diferentes tipos de cimento submetidos a tratamento superficial. 2010. $100 \mathrm{f}$. Dissertação (Mestrado em engenharia civil) Centro de tecnologia do programa de pósgraduação em engenharia civil, Universidade Federal de Santa maria, Santa Maria - RS, 2010.

[7] DOMINICINI, W. K.; CALMON, J. L.. Estudo sobre a modelagem de penetração de cloretos em estruturas de concreto armado. In: $57^{\circ}$ Congresso Brasileiro do Concreto. Bonito MS, IBRACON, 2015.

[8] FELDMAN, R.F. - Significance of porosity measurements on blended cement performance, in: 1st Intern. Conf. on the use of fly ash, silica fume, salg and other mineral by-products in concrete, Montebello, 1983, Malhotra, Ed., ACI, SP-79,1,pp.415-433.

[9] J. LIU, MING-FANG BA, YIN-GANG DU, ZHI-MIN HE, JIAN-BIN CHEN. Effects of chloride ions on carbonation rate of hardened cement paste by X-ray CT techniques. Construction and Building Materials (2016).

[10] J. LIU, Q. QIU, X. CHEN, X. WANG, F. XING, N. HAN. Degradation of fly ash concrete under the coupled effect of carbonation and chloride aerosol ingress, Corrosion Sci. (2016).

[11] MARTÍN-PÉREZ, B. et al. A study of the effect of chloride binding on service life predictions. Cement and Concrete Research, 30, pp. 1215 1223, 2000.

127
[12] MEDEIROS- Junior, R.A.; LIMA, M. G.. Variação da resistividade elétrica do concreto com o formato de corpos de prova para diferentes tipos de cimento, Revista de Engenharia e Tecnologia, v. 7, n. 1,2015

[13] MEDEIROS-JUNIOR, R.A.; LIMA, M.G.; MEDEIROS, M.H.F.; REAL, L.V. Investigação da resistência à compressão e da resistividade elétrica de concretos com diferentes tipos de cimento. Revista ALCONPAT, v.4, n.2, p.116-132, 2014.

[14] MEHTA, P. K; MONTEIRO, P. CONCRETO Estrutura, Propriedades e Materiais. Editora PINI ou Editado pelo IBRACON. 4a Edição, 2014.

[15] MEIJERS, S.J.H. et al. Computational results of a model for chloride ingress in concrete including convection, drying-wetting cycles-and carbonation. Materials and Structures, 38, pp. 145-154, 2005.

[16] OLLIVIER, J.P.; VICHOT, A. Durabilidade do Concreto: Bases Científicas para a formulação de concretos duráveis de acordo com o ambiente. Tradução: O. Cascudo e H. Carasek. São Paulo: IBRACON, 2014.

[17] PEREIRA, Eduardo; MENEGHETTI, Leila Cristina; RESENDE, Andréa; FABRO, Gilmar. Avaliação do grau de corrosão das armaduras para diferentes espessuras de cobrimento. In: CONGRESSO BRASILEIRO DO CONCRETO, 52., 2010, Fortaleza.

[18] PEREIRA, VICTOR CORREIA DE OLIVEIRA; ALMEIDA, KALLINE; MONTEIRO, ELIANA CRISTINA BARRETO. Avaliação da corrosão em argamassas de cimento portland utilizando a técnica de potencial de corrosão. Revista Construindo, Belo Horizonte, v. 4, n. 1, 2012.

[19] RIBEIRO, Daniel Verás. Corrosão em Estruturas de concreto armado: teoria, controle e métodos de análise. Rio de Janeiro: Elsevier, 2014. 